Draft Version March 27, 2017

Preprint typeset using $\mathrm{LT}_{\mathrm{E}} \mathrm{X}$ style emulateapj v. 12/16/11

\title{
SAVE THE PLANET, FEED THE STAR: HOW SUPER-EARTHS SURVIVE MIGRATION AND DRIVE DISK ACCRETION
}

\author{
Jefrrey Fung ${ }^{1}$ \& Eugene Chiang \\ Department of Astronomy, University of California at Berkeley, Campbell Hall, Berkeley, CA 94720-3411 \\ Draft version March 27, 2017
}

\begin{abstract}
Two longstanding problems in planet formation include (1) understanding how planets survive migration, and (2) articulating the process by which protoplanetary disks disperse-and in particular how they accrete onto their central stars. We can go a long way toward solving both problems if the disk gas surrounding planets has no intrinsic diffusivity ("viscosity"). In inviscid, laminar disks, a planet readily repels gas away from its orbit. On short timescales, zero viscosity gas accumulates inside a planet's orbit to slow Type I migration by orders of magnitude. On longer timescales, multiple super-Earths (distributed between, say, 0.1-10 AU) can torque inviscid gas out of interplanetary space, either inward to feed their stars, or outward to be blown away in a wind. We explore this picture with 2D hydrodynamics simulations of Earths and super-Earths embedded in inviscid disks, confirming their slow/stalled migration even under gas-rich conditions, and showing that disk transport rates range up to $\sim 10^{-7} M_{\odot} \mathrm{yr}^{-1}$ and scale as $\dot{M} \propto \Sigma M_{\mathrm{p}}^{3 / 2}$, where $\Sigma$ is the disk surface density and $M_{\mathrm{p}}$ is the planet mass. Gas initially sandwiched between two planets is torqued past both into the inner and outer disks. In sum, sufficiently compact systems of super-Earths can clear their natal disk gas, in a dispersal history that may be complicated and non-steady, but which conceivably leads over Myr timescales to large gas depletions similar to those characterizing transition disks.

Subject headings: accretion, accretion disks - methods: numerical — planets and satellites: formation protoplanetary disks — planet-disk interactions - circumstellar matter — stars: variables: T Tauri, Herbig Ae/Be
\end{abstract}

\section{INTRODUCTION}

Protoplanetary disks have two jobs: make planets and feed their host stars. The first task is frustrated by migration: disk torques force planetary orbits to decay (e.g., Kley \& Nelson 2012), evacuating the very regions where planets are observed in abundance ( $\sim$ 0.1-10 AU from the star; e.g., Clanton \& Gaudi 2014; Burke et al. 2015; Christiansen et al. 2015; Dressing \& Charbonneau 2015). Fulfilling the second task requires a mechanism to transport away the disk's angular momentum. Magnetic torques are promising but depend on seed fields of uncertain provenance (e.g., Bai 2016).

Goodman \& Rafikov (2001) proposed that the two problems are actually one: that planets themselves-if they can survive migration-can provide an effective source of disk viscosity by exciting density waves that transport angular momentum outward. Sari \& Goldreich (2004) emphasized that such planets must be massive enough to open gaps and avoid Type I migration. They focused on giant Jupiter-mass planets, a demographic that is now understood to be rare (e.g., Cumming et al. 2008). In this paper, we turn our attention to super-Earths: bodies of mass $1-10 M_{\oplus}$ that have been discovered by Kepler to be relatively commonplace (e.g., Fressin et al.2013).

Can super-Earths avoid Type I migration? Yes-if their disks are sufficiently inviscid. The dependence of the Type I drift rate on disk viscosity is perhaps under-appreciated, as it is not explicitly called out in the typically quoted Type I formula (see, e.g., Kley \& Nelson 2012). Crucially, without an intrinsic disk viscosity to smooth away the planet's perturbations to the disk's surface density, a pile-up of disk material

email: jeffrey.fung@ berkeley.edu

${ }^{1}$ NASA Sagan Fellow ahead of a migrating planet exerts a "feedback" torque that slows and can even stall migration (Hourigan \& Ward 1984, Ward \& Hourigan 1989). Rafikov (2002b, see his equation 53) calculated that for inviscid disks in which planet-driven waves dissipate by steepening into shocks, the critical planet mass above which Type I migration shuts off is:

$$
M_{\mathrm{cr}} \simeq 4\left(\frac{h_{\mathrm{p}} / r_{\mathrm{p}}}{0.035}\right)^{3}\left(\frac{M_{*}}{M_{\odot}}\right)\left(\frac{\Sigma_{\mathrm{p}} r_{\mathrm{p}}^{2} / M_{*}}{10^{-3}}\right)^{\frac{5}{13}} M_{\oplus},
$$

where $r$ and $h$ are the disk radius and scale height, $\Sigma$ is the disk gas surface density, $M_{*}$ is the central stellar mass, and the subscript $\mathrm{p}$ indicates evaluation near the planet's position. Li et al. (2009) and Yu et al. (2010) have performed numerical simulations supporting the analytic calculations by Rafikov (2002b), and confirming that super-Earths in low-viscosity disks migrate much more slowly (and erratically) than is predicted by Type I.

A planet of mass $M_{\mathrm{p}}$ at $r_{\mathrm{p}}$ drives a disk mass transport rate $\dot{M}$ at distance $r$ of

$$
\dot{M}(r)=-\frac{2 F_{0}}{l} r \frac{\partial \varphi(r)}{\partial r}
$$

where

$$
F_{0}=\Sigma_{\mathrm{p}} r_{\mathrm{p}}^{2} l_{\mathrm{p}} \Omega_{\mathrm{p}}\left(\frac{M_{\mathrm{p}}}{M_{*}}\right)^{2}\left(\frac{h_{\mathrm{p}}}{r_{\mathrm{p}}}\right)^{-3}
$$

measures the total angular momentum carried away per time by planet-driven waves (a.k.a. the total integrated one-sided Lindblad torque), $\Omega$ is the orbital frequency, and $l=\Omega r^{2}$ is the specific angular momentum. The dimensionless function $\varphi(r)$ describes how waves, as they travel away from the planet, damp with distance, depositing their angular momentum to disk gas and thereby propelling material radially. From 
Rafikov (2002b, see his equations B1 and 33),

$$
\varphi \sim\left(\frac{M_{\mathrm{p}}}{M_{\text {thermal }}}\right)^{-\frac{1}{2}}\left(\frac{\left|r_{\mathrm{p}}-r\right|}{h_{\mathrm{p}}}\right)^{-\frac{5}{4}}
$$

valid for $M_{\mathrm{p}} \lesssim M_{\text {thermal }} \equiv\left(h_{\mathrm{p}} / r_{\mathrm{p}}\right)^{3} M_{*}$ and $\varphi \lesssim 1$ (i.e., distances far enough from the planet that the waves are dissipating in weak shocks), and where we have ignored order-unity constants and all radial variations in $h, \Sigma$, and gas sound speed. It follows that

$$
\dot{M}(r) \sim \operatorname{sign}\left(r-r_{\mathrm{p}}\right) \Sigma_{\mathrm{p}} r_{\mathrm{p}}^{2} \Omega_{\mathrm{p}}\left(\frac{M_{\mathrm{p}}}{M_{*}}\right)^{\frac{3}{2}}\left(\frac{h_{\mathrm{p}}}{r_{\mathrm{p}}}\right)^{-\frac{5}{2}}\left(\frac{\left|r_{\mathrm{p}}-r\right|}{h_{\mathrm{p}}}\right)^{-\frac{9}{4}} .
$$

At $r<r_{\mathrm{p}}, \dot{M}$ as given by Equation $(5)$ is negative (mass flows inward), and vice versa; a planet tends to repel material away from itself. To avoid minus signs, we will ignore this formal sign convention so that all our reported values for $\dot{M}$ will be positive and understood to be inward unless otherwise indicated.

Note how $\dot{M} \propto M_{\mathrm{p}}^{3 / 2}$ and not $M_{\mathrm{p}}^{2}$. Although the total Lindblad torque scales as $M_{\mathrm{p}}^{2}$ (Equation 3, that torque is distributed over a distance that increases with decreasing $M_{\mathrm{p}}$ (as $M_{\mathrm{p}}^{-2 / 5}$, as can be seen by solving for $\left|r_{\mathrm{p}}-r\right|$ in terms of $M_{\mathrm{p}}$ at fixed $\varphi$ in Equation 4). Thus at fixed distance away from the planet, $\dot{M}$ increases with $M_{\mathrm{p}}$ with a power less than 2 .

Inserting $M_{\mathrm{p}}=10 M_{\oplus}$ and other nominal parameters (for $r<r_{\mathrm{p}}$ ) into $(5)$ yields

$$
\begin{aligned}
\dot{M}(r) \sim 10^{-8} & \left(\frac{\Sigma_{\mathrm{p}} r_{\mathrm{p}}^{2}}{10^{-3} M_{\odot}}\right)\left(\frac{2 \pi / \Omega_{\mathrm{p}}}{1 \mathrm{yr}}\right)^{-1}\left(\frac{M_{\mathrm{p}} / M_{*}}{3 \times 10^{-5}}\right)^{\frac{3}{2}}\left(\frac{h_{\mathrm{p}} / r_{\mathrm{p}}}{0.035}\right)^{-\frac{1}{4}} \\
& \left(\frac{\left(r_{\mathrm{p}}-r\right) / r_{\mathrm{p}}}{0.5}\right)^{-\frac{9}{4}} M_{\odot} \mathrm{yr}^{-1},
\end{aligned}
$$

comparable to accretion rates measured for classical $\mathrm{T}$ Tauri stars (e.g., Calvet et al. 2005, Hartmann et al. 2006; SiciliaAguilar et al. 2010; Ingleby et al. 2013). Note how weakly $M$ depends on $h_{\mathrm{p}} / r_{\mathrm{p}}$, underscoring how $\dot{M}$ does not scale simply as the total Lindblad torque in Equation 3 (which scales as $\left.\left(h_{\mathrm{p}} / r_{\mathrm{p}}\right)^{-3}\right)$, but depends also on the distance over which that torque is exerted, as we have described above.

The above considerations indicate that with super-Earths we might have our cake (survive migration) and eat it, too (drive disk accretion). Of course, a single super-Earth is insufficient because its reach is too short ( $\dot{M}$ drops as $\left.\left|r-r_{\mathrm{p}}\right|^{-9 / 4}\right)$. Multiple super-Earths are needed to shuttle the accretion flow from distances of a few AU down to the stellar radius. Reality will be non-steady and likely messy (see, e.g., Figure 4 of Rafikov 2002a), with material between adjacent planets having a fate that is not obvious: does the sandwiched gas drain inward, or does the inner planet hold back material pushed inward by the outer planet? And to what extent do super-Earths migrate with the accretion flow they drive?

Here we explore these questions using fully non-linear, 2D hydrodynamical simulations of super-Earths embedded in inviscid disks. We measure the migration histories $r_{\mathrm{p}}(t)$ and accretion rates $\dot{M}$ in simulations containing 1 or 2 super-Earths, experimenting with varying the disk surface density and the planet mass to test Equation 6 Our numerical methods are given in Section 2. Results are presented in Section 3 and placed into broader context in Section 4.

\section{NUMERICAL METHOD}

We use the graphics processing unit (GPU) accelerated hydrodynamics code PEnGUIn (Fung 2015) to perform 2D simulations of disk-planet interactions. It is a Lagrangianremap shock-capturing code that uses the piecewise parabolic method (Colella \& Woodward 1984) to solve the continuity and momentum equations:

$$
\begin{aligned}
& \frac{\mathrm{D} \Sigma}{\mathrm{D} t}=-\Sigma(\nabla \cdot \mathbf{v}), \\
& \frac{\mathrm{D} \mathbf{v}}{\mathrm{D} t}=-\frac{1}{\Sigma} \nabla p+\frac{1}{\Sigma} \nabla \cdot \mathbb{T}-\nabla \Phi,
\end{aligned}
$$

where $\Sigma$ is the gas surface density, $\mathbf{v}$ the velocity field, $p$ the vertically averaged gas pressure, $\mathbb{T}$ the Newtonian stress tensor, and $\Phi$ the combined gravitational potential of the star and the planet(s). We use a globally isothermal equation of state: $p=c_{\mathrm{s}}^{2} \Sigma$ with a spatially constant sound speed $c_{\mathrm{s}}=0.035 v_{\mathrm{K}, 1 \mathrm{AU}} \simeq 1 \mathrm{~km} \mathrm{~s}^{-1}$ where $v_{\mathrm{K}, 1 \mathrm{AU}}$ is the Keplerian velocity at $1 \mathrm{AU}$ around a $1 M_{\odot}$ star. (This $c_{\mathrm{S}}$ corresponds to a disk temperature of $300 \mathrm{~K}$ assuming a mean molecular weight of 2.34.)

In a polar coordinate system (radius $r$, azimuth $\phi$ ) centered on the star,

$$
\begin{aligned}
\Phi & =-\frac{G M_{*}}{r}+\sum_{i=1}^{N_{\mathrm{p}}} \Phi_{\mathrm{p}, i} \\
\Phi_{\mathrm{p}, i} & =-\frac{G M_{\mathrm{p}, i}}{\sqrt{r^{2}+r_{\mathrm{p}, i}^{2}-2 r r_{\mathrm{p}, i} \cos \phi_{i}^{\prime}+r_{\mathrm{s}, i}^{2}}}+\frac{G M_{\mathrm{p}, i} r \cos \phi_{i}^{\prime}}{r_{\mathrm{p}, i}^{2}}
\end{aligned}
$$

where $G$ is the gravitational constant, $M_{*}=1 M_{\odot}$ is the stellar mass, the subscript $i$ labels each planet, $N_{\mathrm{p}}$ is the total number of planets, $M_{\mathrm{p}}$ is the planet mass, $\Phi_{\mathrm{p}}$ the planet's gravitational potential, $r_{\mathrm{p}}$ the planet's radial coordinate, $r_{\mathrm{s}}$ the smoothing length of the planet's potential, and $\phi^{\prime}=\phi-\phi_{\mathrm{p}}$ the azimuthal separation from the planet. The stress tensor $\mathbb{T}$ is proportional to the kinematic viscosity $v$. Most of our simulations are of inviscid disks with $v=0$. For our viscous disk simulations, we use $v=\alpha c_{\mathrm{s}} h$, where the Shakura-Sunyaev parameter $\alpha=0.001, h=c_{\mathrm{s}} / \Omega_{\mathrm{K}}$ is the local scale height, and $\Omega_{\mathrm{K}}=\sqrt{G M_{*} / r^{3}}$ is the Keplerian orbital angular frequency. At $r=1 \mathrm{AU}, h / r=0.035$. We set $r_{\mathrm{s}}=0.5 h$, as is appropriate for 2D simulations (Müller et al.2012).

A given planet feels the gravitational force from the star, the disk, and other planets. The disk force on the planet is calculated by direct summation over all mass elements in the disk, with the "background" axisymmetric component of the disk surface density subtracted off. Because the disk does not feel its own gravity at all (i.e., we ignore disk self-gravity; see equation 9), eliminating this axisymmetric component in the disk-planet forcing improves consistency between the motions of the planets and the disk. Planet migration should be minimally affected by this procedure, since the background component of $\Sigma$ exerts no torque. Spurious forces arising from within the planet's Hill sphere are sometimes a concern if this region is under-resolved. The Hill radius, $r_{\mathrm{H}}=\left(M_{\mathrm{p}} / 3 M_{*}\right)^{1 / 3}$, ranges from 0.3 to $0.6 h_{\mathrm{p}}$, similar to the smoothing length $r_{\mathrm{s}}$. We have verified that the torque generated within a radius of $0.5 r_{\mathrm{H}}$ from the planet is negligible, and so we do not excise the Hill sphere in force calculations. The planets' motions are integrated using a kick-drift-kick leapfrog scheme, with 
the drift step occurring synchronously with the hydrodynamics step; i.e., the planets' positions are linear in time within a hydrodynamics step.

\subsection{Initial and boundary conditions, and grid parameters}

Table 1 lists the parameters used by our 6 models. The disk is initialized with a power-law surface density:

$$
\Sigma=\Sigma_{0}\left(\frac{r}{\mathrm{AU}}\right)^{-\frac{3}{2}} \text {. }
$$

We consider both gas-rich disks having $\Sigma_{0}=8.5 \times 10^{3} \mathrm{~g} \mathrm{~cm}^{-2}$ resembling the minimum-mass extrasolar nebula (Chiang \& Laughlin 2013), and gas-poor disks having a surface density $1000 \times$ lower. The initial velocity field is axisymmetric and Keplerian, with corrections from gas pressure:

$$
\Omega=\sqrt{\Omega_{\mathrm{K}}^{2}+\frac{1}{r \Sigma} \frac{\mathrm{d} p}{\mathrm{~d} r}} .
$$

One planet, whose mass is increased gradually over the first $10 \mathrm{yr}$ of the simulation to the full value of $M_{\mathrm{p}}$ (either 1,3 , or $10 M_{\oplus}$ ), is placed initially at $r=r_{\mathrm{p}, 1}$ (either 1 or $0.75 \mathrm{AU}$ ) and $\phi_{\mathrm{p}, 1}=\pi$. In two-planet models, we place a second planet of equal mass to the first at $r=r_{\mathrm{p}, 2}$ (either 1.2 or $1.05 \mathrm{AU}$ ) and $\phi_{\mathrm{p}, 2}=\pi$ initially.

Our simulation grid spans the full $2 \pi$ in azimuth, and extends from an outer radius of $1.8 \mathrm{AU}$ to an inner radius $r_{\text {in }}$ that equals either 0.4 or $0.3 \mathrm{AU}$ depending on whether $r_{\mathrm{p}, 1}=1$ AU or 0.75 AU (see Table 1). Grid dimensions are $800(r) \times$ $3200(\phi)$ when $r_{\text {in }}=0.4 \mathrm{AU}$, and $960 \times 3200$ when $r_{\text {in }}=0.3$ AU. Cells are spaced logarithmically in radius and uniformly in azimuth. Our choices yield a resolution of $\sim 18$ cells per scale height $h$ in both directions at $r=1 \mathrm{AU}$ (similar to the resolution of $\mathrm{Li}$ et al. 2009). Simulations at twice our standard resolution did not produce significant changes in either planet migration or disk accretion rate for the first $100 \mathrm{yr}$. We also tested our inviscid disk model without a planet, and found that the numerical noise in $|\dot{M}|$ was about 3 orders of magnitude below planet-driven disk accretion rates, corresponding to a numerical viscosity of $\alpha<10^{-5}$.

Radial boundary conditions require special care in this study. After experimenting with a few ways to measure disk accretion rates, we found that the most stable method was to track the total disk mass within a cylinder of radius $0.6 \mathrm{AU}-$ a distance intermediate between the innermost planet and the inner disk boundary - while preventing mass from leaving the grid. We adopt "zero flux" boundary conditions where mass and momentum fluxes across the inner and outer disk edges are always zero. In PEnGUIn, this is achieved by solving a special Riemann problem at the boundaries, one where no wave travels toward the simulation domain, and where the radial velocity outside the domain is always zero. This implementation conserved the total mass within the simulation domain to numerical accuracy. The accretion rate $\dot{M}$ at $r=0.6$ $\mathrm{AU}$ is calculated by following over time the disk mass enclosed, $M_{0.6 \mathrm{AU}}(t)$. Because the function $M_{0.6 \mathrm{AU}}(t)$ fluctuates strongly, we fit independent lines to segments of data each lasting $20 \mathrm{yr}$, taking $\dot{M}$ from the best-fitting slopes.

As a planet repels material away from its orbit, our boundary conditions result in gas piling up at the inner and outer

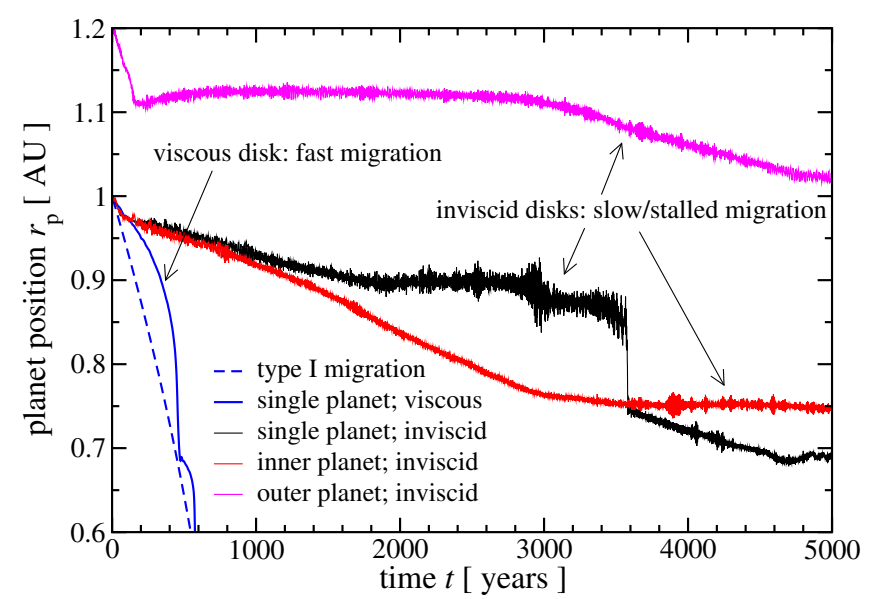

FIG. 1.- Type I migration can be defeated for super-Earths in inviscid disks. Shown here are the orbital radii vs. time of planets in three of our simulations: a single $10 M_{\oplus}$ planet in a gas-rich $\left(\Sigma_{0}=8.5 \times 10^{3} \mathrm{~g} \mathrm{~cm}^{-2}\right)$ viscous $\left(\alpha=10^{-3}\right)$ disk (blue, model \#1); a single planet of the same mass in a gas-rich inviscid $(\alpha=0)$ disk (black, model \#2); and two such planets in a gas-rich inviscid disk (red+magenta, model \#3). The blue dashed line is the theoretically expected trajectory from Type I migration (the integral of Equation 13 with $C=2$ ).

boundaries. Our results can only be trusted to the extent that these boundary pile-ups do not interfere with planet migration and disk accretion. We therefore limit ourselves to studying only the first few thousand years of planet-disk interactions, before boundary effects become too large.

\section{RESULTS}

We assess to what extent planets migrate in inviscid disks $(\$ 3.1)$, and study how planet-driven accretion rates evolve with time and depend on disk and planet masses $(\$ 3.2)$. For planet migration, models \#1-3 demonstrate differences between viscous and inviscid disks, and between single-planet and two-planet systems. For planet-driven accretion, we vary disk and planet masses in models \#3-6 to test Equation 6.

\subsection{Planet migration}

Figure 1 plots the orbital evolution of planets, each of mass $10 M_{\oplus}$, in the gas-rich disk models (\#1-3). Overplotted for comparison is the trajectory expected from integrating the Type I migration rate,

$$
\dot{r}_{\mathrm{p}, \text { Type I }}=-2 C r_{\mathrm{p}} \Omega_{\mathrm{p}}\left(\frac{\Sigma_{\mathrm{p}} r_{\mathrm{p}}^{2}}{M_{\mathrm{p}}}\right)\left(\frac{M_{\mathrm{p}}}{M_{*}}\right)^{2}\left(\frac{h_{\mathrm{p}}}{r_{\mathrm{p}}}\right)^{-2},
$$

using the unperturbed surface density law in Equation 11 to evaluate $\Sigma_{\mathrm{p}}$ (using the actual surface density in the viscous disk simulation \#1 would give practically identical results, since gaps do not form in that model). Three-dimensional simulations suggest that $C \sim 2-3$ for our given disk profile (D’Angelo \& Lubow 2010; Fung et al. 2017); the blue dashed curve in Figure 1 uses $C=2$. Figure 2 displays azimuthally averaged surface density profiles at various epochs in gas-rich, inviscid disk models \#2 and \#3, with planet locations marked.

In agreement with the simulations of Li et al. (2009) and Yu et al. (2010), the planet migration rate in our viscous disk (model \#1) is similar to the Type I rate (punctuated by what appear to be episodes of even faster Type III migration; Masset \& Papaloizou 2003, Peplinski 2008), and much slower in inviscid disks (models \#2 and \#3). The initially rapid migration seen in the inviscid simulations at $t \lesssim 200 \mathrm{yr}$ is a transient that decays after disk surface densities adjust to plan- 
TABLE 1

Model Parameters

\begin{tabular}{cccccccc}
\hline \hline Model \# & $M_{\mathrm{p}}\left(M_{\oplus}\right)$ & $r_{\mathrm{p}, 1}(\mathrm{AU})$ & $r_{\mathrm{p}, 2}(\mathrm{AU})$ & $\Sigma_{0}\left(\mathrm{~g} \mathrm{~cm}^{-2}\right)$ & $\alpha$ & $r_{\text {in }}(\mathrm{AU})$ & $t_{\text {end }}$ (years) \\
\hline 1 & 10 & 1 & - & $8.5 \times 10^{3}$ & $10^{-3}$ & 0.4 & 700 \\
2 & 10 & 1 & - & $8.5 \times 10^{3}$ & 0 & 0.4 & 5000 \\
3 & 10 & 1 & 1.2 & $8.5 \times 10^{3}$ & 0 & 0.4 & 5000 \\
4 & 10 & 0.75 & 1.05 & 8.5 & 0 & 0.3 & 2000 \\
5 & 3 & 0.75 & 1.05 & 8.5 & 0 & 0.3 & 2000 \\
6 & 1 & 0.75 & 1.05 & 8.5 & 0 & 0.3 & 2000 \\
\hline
\end{tabular}

Note. $-t_{\text {end }}$ is the end time of a simulation, in units where the Keplerian orbital period at 1 $\mathrm{AU}$ is 1 year. Also, $r_{\mathrm{p}, 1}$ and $r_{\mathrm{p}, 2}$ are merely the initial planet locations at $t=0$; the planets are completely free to migrate in the simulations.
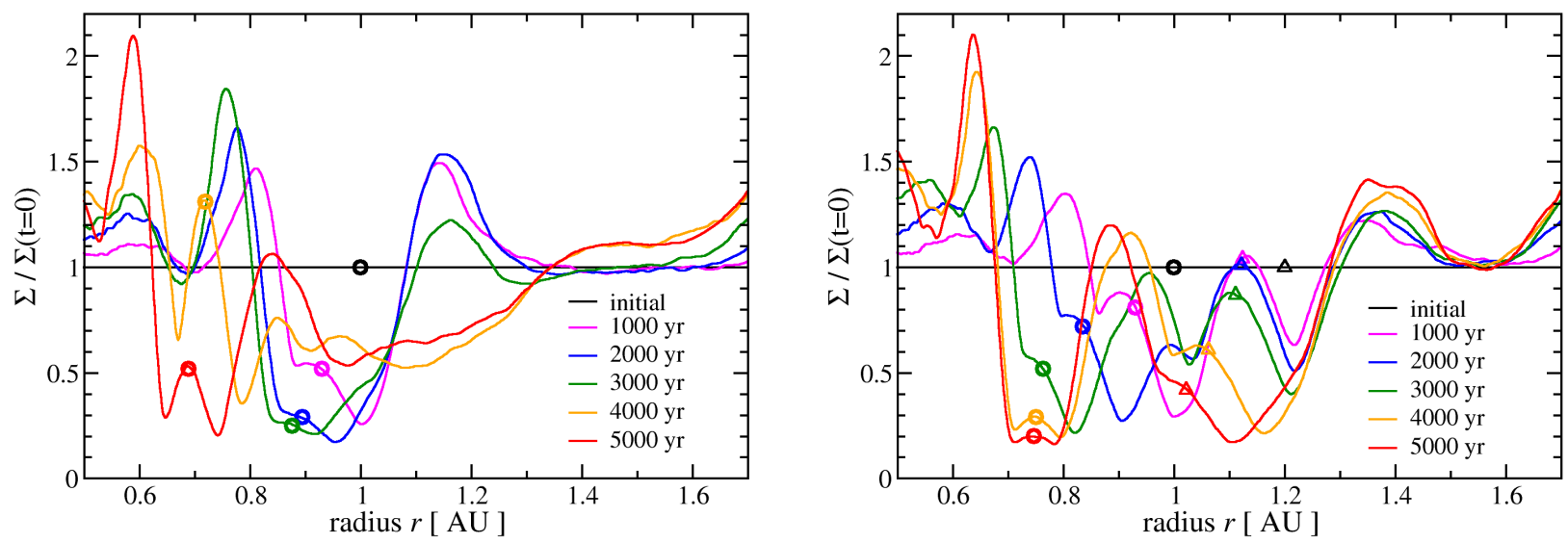

Fig. 2.- Left: Surface density profiles, azimuthally averaged and normalized against the initial power-law profile, measured at 1000-yr intervals for our singleplanet, inviscid, gas-rich disk model (\#2). Right: Analogous profiles for our two-planet, inviscid, gas-rich disk model (\#3). Circles (triangles) mark the locations of the inner (outer) planet.

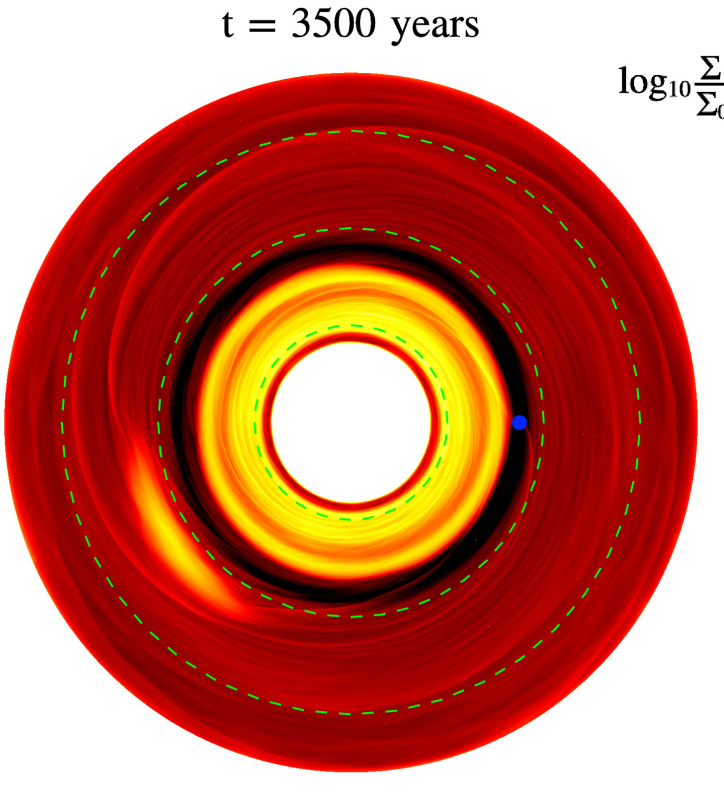

FIG. 3.- Surface density snapshot of our single-planet, gas-rich simulation (model \#2) at 3500 years. Green dashed circles are at $0.5,1$, and $1.5 \mathrm{AU}$. The blue dot indicates the planet's position. This snapshot is taken near the time of the planet-vortex close encounter. The vortex, of approximate mass 100 $M_{\oplus}$, is seen at around 1.1 AU. After the encounter, the planet is perturbed radially inward (Figure 1). The vortex ultimately decays away (Figure 4). etary Lindblad torques, i.e., after the surface density pile-up ahead of the planet attains a fractional amplitude on the order of unity. After this initial adjustment period, migration slows and even stalls at times, with radial positions changing by $\sim 10-30 \%$, or less, over kyr timescales.

In the single-planet, gas-rich simulation (black curve in Figure 1. model \#2), the planet journeys slowly inward for the first $\sim 2000 \mathrm{yr}$ and practically stops in the mean from $t \simeq 2000-3500 \mathrm{yr}$, as disk gas that the planet has pushed inward to $r \simeq 0.7-0.8$ AU piles up (blue and green curves in the left panel of Figure 2 and stymies further migration. At $t \simeq 3500 \mathrm{yr}$, the planet experiences a sudden drop in orbital radius; we traced this drop to a close encounter between the planet and a vortex formed at its outer gap edge at $r \simeq 1.1$ AU (see Figure 3). Some time after the encounter, the vortex gradually disperses, completely decaying away by the end of our simulation at $5000 \mathrm{yr}$ (Figure 4, left panel). Similar planet-vortex interactions were found in simulations by Lin \& Papaloizou (2010) and Yu et al. (2010). Thereafter, the planet's migration returns to its near-zero mean pace.

In our two-planet, gas-rich simulation (\#3), the outer planet stalls for the first $\sim 3000 \mathrm{yr}$ (magenta curve in Figure 1), apparently trapped at a local surface density maximum created by the inner planet whose forcing dominates: see the magenta curve in the right panel of Figure 2, and note how similar it is to the corresponding magenta curve in the left panel for the single-planet case. Gas pushed inward by the outer planet strengthens the torque on the inner planet and forces the latter to migrate inward by $\sim 20 \%$ over the same time period (red curve in Figure 1); contrast this behavior with the stalling ob- 
model 2

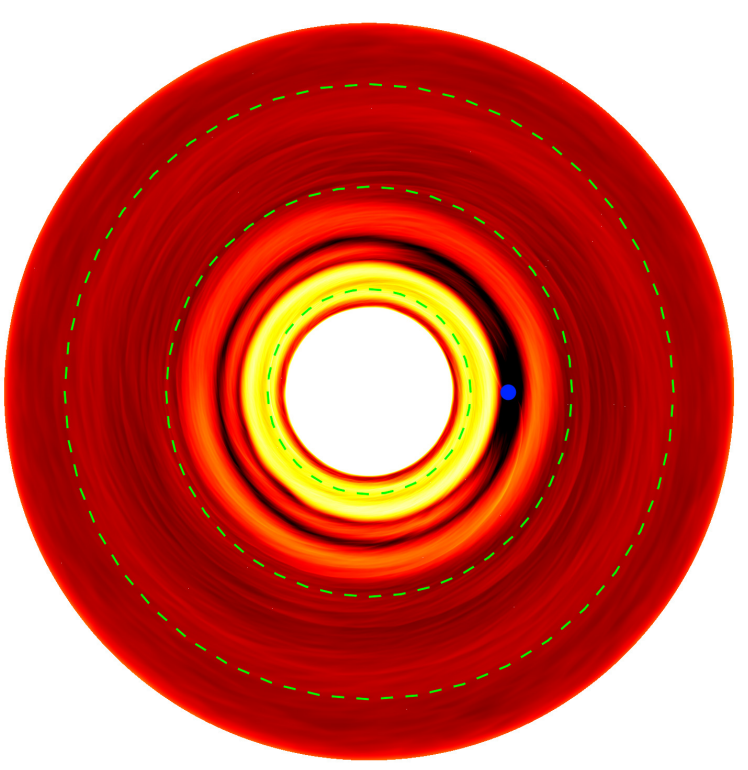

model 3

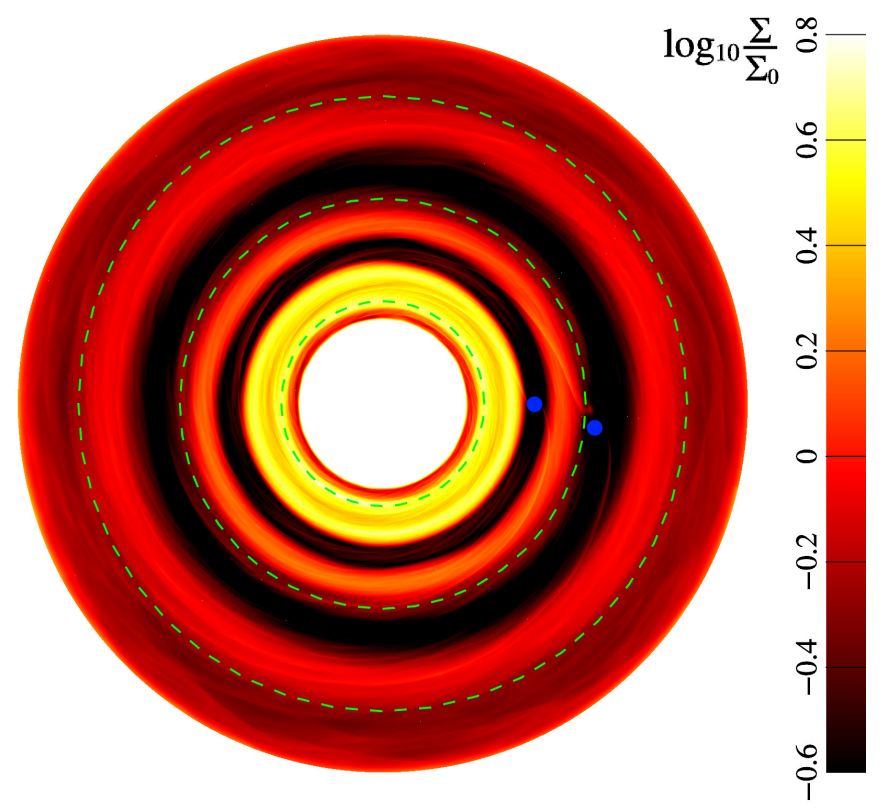

FIG. 4.- Surface density snapshots of our inviscid gas-rich simulations, for our single-planet model (\#2, left) and two-planet model (\#3, right) cases, taken at their end time of 5000 years. Green dashed circles are at $0.5,1$, and $1.5 \mathrm{AU}$. The blue dots indicate the planets' positions. In the single-planet snapshot, note that the vortex seen in Figure 3 has completely decayed away. No comparably strong vortex was found in the two-planet simulation at any time, and the density field appears more axisymmetric than in the single-planet case.

served in the single-planet case (black curve). Eventually, at $t \sim 4000 \mathrm{yr}$, the outer planet disperses the surface density maximum in its vicinity, and proceeds to migrate slowly inward, slowing down near $t \sim 5000 \mathrm{yr}$ as it runs into sandwiched gas. Meanwhile, the inner planet ultimately comes to a near halt in much the same way that it does in the singleplanet simulation, having run into material that has piled up just interior to its orbit. The right panel of Figure 4 shows the final surface density distribution. We emphasize that this pileup is physical as it is located at $r \simeq 0.6 \mathrm{AU}$, away from the inner grid boundary of the simulation at $r_{\text {in }}=0.4 \mathrm{AU}$. The latter location has its own separate pile-up, which does not grow to significance over the limited duration of our simulations. The same statement applies to the outer grid boundary.

We note that in none of the two-planet simulations did we observe the formation of a vortex like the one seen in our single-planet simulation. This difference might be physical, and deserves attention in future studies of planet-vortex interactions.

All other factors being equal, lower disk masses should lead to even slower planetary migration rates. This is confirmed in our gas-poor simulations (models \#4-6) which exhibit no measurable change in planet mean radial positions. Thus the gas-poor simulations can be used to diagnose disk accretion rates without the complicating effects of planetary migration, as we discuss in the next subsection.

\subsection{Disk accretion}

Figure 5 shows disk accretion rates as functions of time for all our inviscid models. As described in $\$ 2.1$ the accretion rate $\dot{M}$ is measured by tracking the build up of disk mass inside $r=0.6 \mathrm{AU}$, a location interior to the planets at all times. Later, in $\$ 3.2 .1$, we track the movement of mass initially between two planets.

We begin by checking whether our simulations are compatible with the analytic expectation for $\dot{M}$ given by Equation 6 .
The comparison is best made at early times of the simulation, $t \lesssim 1000 \mathrm{yr}$, before radial surface density profiles become too distorted. For the single-planet, gas-rich model \#2, Equation 6 yields $\dot{M} \sim 3 \times 10^{-8} M_{\odot} \mathrm{yr}^{-1}$. This prediction is within a factor of $\sim 2$ of the simulated result at early times (left panel of Figure 5). As for the corresponding two-planet model \#3, we expect from (6) that $\dot{M}$ should be only fractionally larger than for the single-planet case; the second planet is farther removed from where we measure $\dot{M}(r=0.6 \mathrm{AU})$, and so makes only a $\sim 50 \%$ contribution to the accretion flow there as compared to the inner planet. This is approximately consistent with Figure 5.

For our gas-poor model \#4, Equation 6 predicts $\dot{M} \sim 1.5 \times$ $10^{-10} M_{\odot} \mathrm{yr}^{-1}$, again within a factor of 2 of the simulated result (right panel of Figure 5). Scaling the planet mass $M_{\mathrm{p}}$ down by a factor of 10 from models \#4 to \#6 should, according to Equation 6, reduce $\dot{M}$ by a factor of $10^{3 / 2} \simeq 30$. By comparison, Figure 5 shows a factor of $\sim 20$ decrease between these two models; we consider this acceptable agreement with the analytic expectation. In summary, our simulations support the various functional dependencies predicted by Equation 6 to within a factor of 2 .

At later times, $t \gtrsim 1000 \mathrm{yr}$, we observe time variability in $\dot{M}$ caused by the deepening of planetary gaps, and by planet migration. These variations are limited to factors of a few. The simulations easiest to interpret are models \#4-6 (right panel of Figure 5 which have too little disk gas to drive planet migration. The initial gradual decline in $\dot{M}$ seen in model \#4 is caused by the deepening of gaps opened by its $10-M_{\oplus}$ planets; over the course of $2000 \mathrm{yr}$, the gas density in the immediate vicinity of the planets decreases by a factor of $\sim 5$ for the inner planet and by a factor of $\sim 3$ for the outer one. Models \#5 and \#6 exhibit steadier accretion rates, as their planets have lower masses which are less effective at opening gaps.

More complicated behavior is seen in the gas-rich simulations where planets migrate more appreciably. Comparison of 

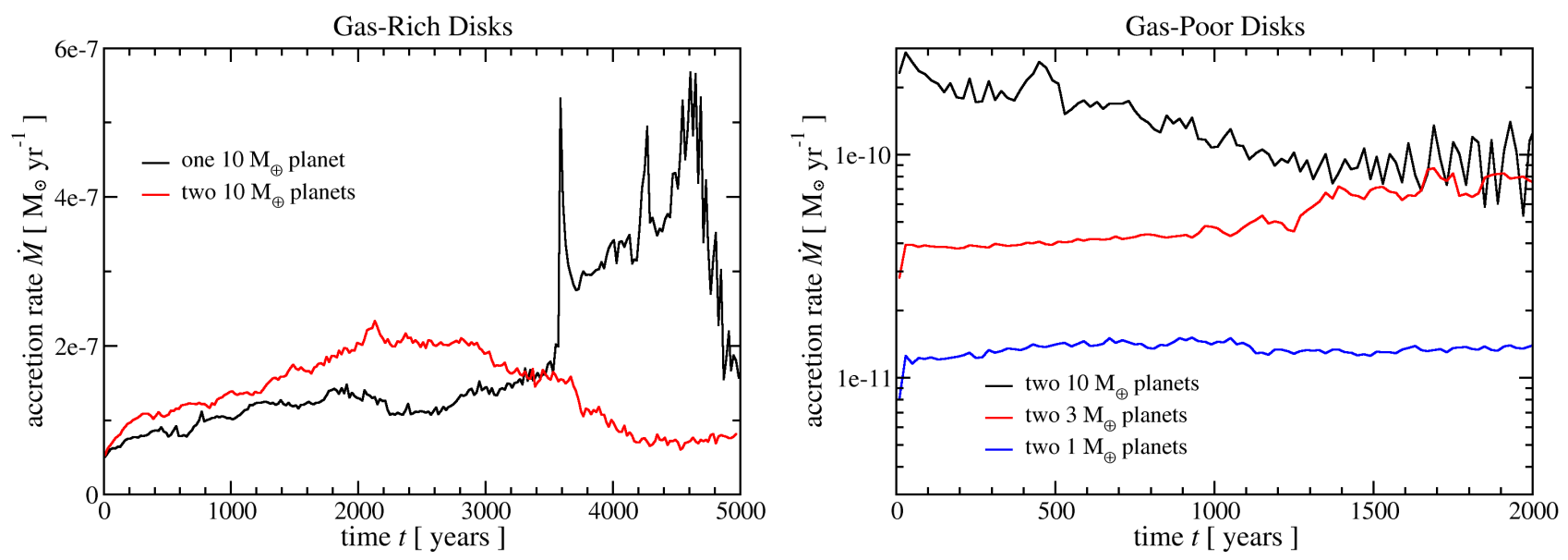

FIG. 5.- Disk accretion rates measured at $r=0.6 \mathrm{AU}$, a location interior to all planets at all times. Each data point represents an average over 20 years (see $\$ 2.1$. Left: Gas-rich $\alpha=0$ simulations (model \#2 in black, \#3 in red). Right: Gas-poor $\alpha=0$ models (\#4-6 in black, red, and blue, respectively). At $t \lesssim 1000$ yr, accretion rates agree with predictions from Equation 6 to within a factor of 2. Variations in $\dot{M}$ at later times reflect planet migration (compare with Figure 1 ) and deepening of gaps.

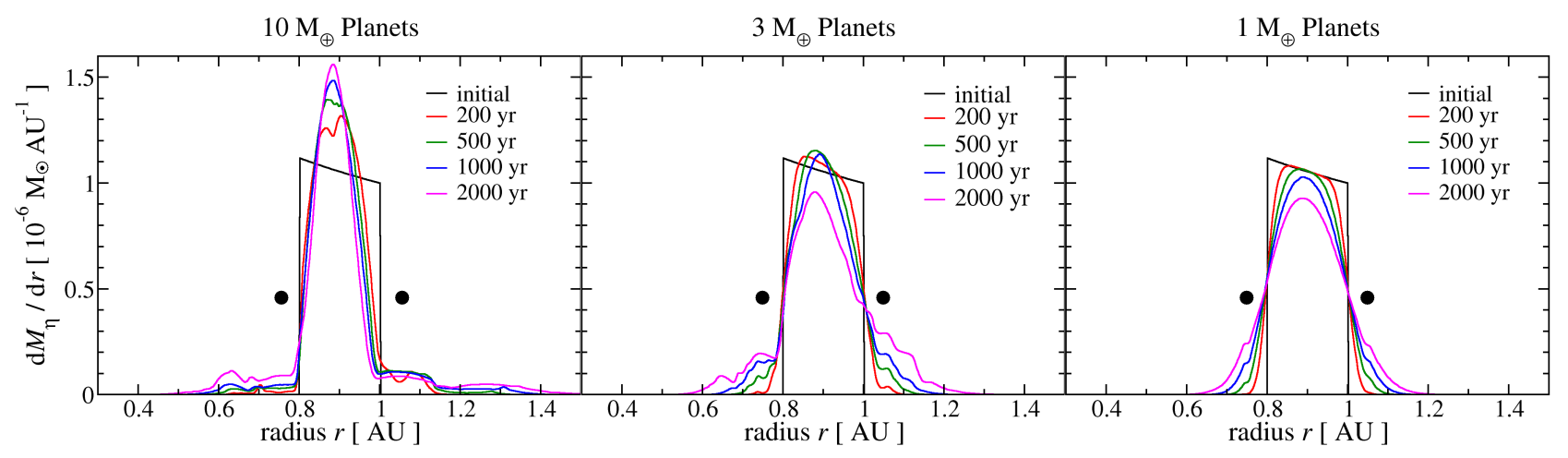

Fig. 6. - Tracking the material initially sandwiched by two planets. From left to right, we show results from models \#4, 5, and 6. Black circles denote the planets' locations which do not change in these gas-poor runs. In all cases, the gas escapes over time to either side of the planetary pair.

Figures 1 and 5 reveals that increases in $\dot{M}$ can be traced to planets moving inward, either gradually, as in the first 2000 yr of models \#2 and \#3, or suddenly, as in the planet-vortex encounter at $t \simeq 3300 \mathrm{yr}$ in model \#2. Decreases in $\dot{M}$ correspond to planets opening gaps upon moving to new locations.

\subsubsection{The fate of gas initially sandwiched between planets}

Disk accretion driven by planets would be impractical if material residing between planets were unable to escape. We track this sandwiched gas in simulations \#4-6, each containing a pair of planets which migrate negligibly. We assign each gas parcel a "passive scalar" $\eta$ that equals 1 for gas initially located between $r=0.8$ and $1 \mathrm{AU}$ (between the two planets), and is 0 everywhere else. Gas elements carry $\eta$ as a conservative quantity.

Figure 6 follows the $\eta$-tagged gas by plotting

$$
\frac{\partial M_{\eta}}{\partial r}=\int_{0}^{2 \pi} \eta \Sigma r d \phi,
$$

vs. $r$ at various times. We find that the sandwiched gas is torqued both inward and outward, escaping in roughly equal amounts to the inside of the inner planet and to the outside of the outer planet. The opposing torques from the two planets do not in general cancel. The opposing torques from the two planets do not in general cancel, although in model \#4 containing the highest mass planets, some gas does concentrate along the midline between the planets in a "shepherded" ring, resulting in less mass leaking out of the sandwiched region. Looking at models \#4-6 in Figure 6, we see no clear trend between the rate at which sandwiched gas escapes and planet mass. There seems to be a complicated confluence of effects in the sandwiched region. Lindblad torques act to shepherd some gas while also opening gaps that reduce the local gas density; and co-orbital torques allow gas to escape via horseshoe orbits, whose libration times scale only weakly with planet mass $\left(t_{\mathrm{lib}} \propto M_{\mathrm{p}}^{-1 / 2} ;\right.$ Paardekooper \& Papaloizou 2009). A detailed analysis is deferred to another paper; for now, we conclude that, at least for comparable mass planets with orbital spacings like the one we have assumed, the inner planet presents a porous barrier to material pushed inward by the outer planet. Apparently gas that is pushed by the outer planet toward the inner planet can be shuttled past the latter on horseshoe orbits (and vice versa).

\section{SUMMARY AND DISCUSSION}

Using hydrodynamical simulations, we have demonstrated that super-Earths in inviscid disks can simultaneously avoid type I migration (Figure 1) and promote disk accretion (Figure 5 by driving density waves. Disk accretion rates mea- 
sured from our simulations verify analytic predictions (Equation 6) to within a factor of 2 . We observed gap opening and planet migration in inviscid disks to be modest and to introduce order-unity effects on the disk accretion rate. We also found in our two-planet simulations that material initially sandwiched between two planets leaks past both into the innermost and outermost disks (Figure 6).

Our models omit a number of effects. Many of these are not overly concerning. Although our simulations are 2D, no substantive difference between $2 \mathrm{D}$ and 3D treatments of planet-disk interactions in viscous disks has been reported visà-vis gap opening (Fung \& Chiang 2016) or planetary torques (Fung et al. 2017). Our neglect of disk self-gravity should be an excellent approximation, as the Toomre $Q$-values of our disks greatly exceed unity. Our planets are not allowed to accrete gas, but super-Earths/sub-Neptunes are inferred observationally to have only modest amounts of gas-less than $10 \%$ by mass-acquired gradually over the entire disk lifetime (Lee \& Chiang 2016).

More interesting frontiers to pursue include incorporating disk thermodynamics, as radiative cooling and differential heating across gap walls are thought to materially affect planet-disk interactions (e.g., Kley \& Nelson 2012, Tsang et al. 2014). Of course, extending the durations of the simulations, and including more planets with different orbital architectures, would also be welcome, for greater realism and to enable more direct connections with observations. Closer study of gap depths is warranted; we observed surface density contrasts only on the order of unity (Figure 2), in clear deviation from scaling relations derived from viscous disks (e.g., Fung et al. 2014), and surprising insofar as less viscous gas should be less effective at diffusively back-filling gaps. The shallowness of the gaps is due partly to the planets migrating and re-starting the gap-opening process at each new radial location (Malik et al. 2015). But how much of it is due to the limited duration of our simulations $(\leq 5000 \mathrm{yr})$, or to hydrodynamical instabilities like the Rayleigh instability (Fung \& Chiang 2016), remains to be worked out. Finally, survival against planetary migration is not guaranteed: planets with mass $M_{\mathrm{p}}>M_{\mathrm{cr}}$ (Equation 1) stall but less massive planets do not. The question is whether rocky planets can coagulate fast enough to cross the $M_{\mathrm{cr}}$ threshold before they succumb to migration.

Our results support the proposal by Goodman \& Rafikov (2001) that predominantly rocky planets-super-Earths and Earths - can solve, or at least help to solve, the problem of how protoplanetary gas disks ultimately disperse. Given that a single planet can push gas over a lengthscale of approximately half its orbital radius, shuttling gas from 5 AU down to 0.1 AU would require about 6 super-Earths distributed in roughly equal logarithmic intervals across this distance. Such planet multiplicities are reasonable, given the profusion of superEarths/sub-Neptunes discovered by Kepler (e.g., $\mathrm{Pu} \& \mathrm{Wu}$ 2015). To be sure, a disk accretion flow driven by a planetary system will be unsteady, changing not only on secular, Myrlong timescales, but also on much shorter ones, with mass alternately accumulating and dispersing in interplanetary space, as we have seen in our simulations (Figure 2). No matter how complicated the history, however, all gas must ultimately be torqued out of sufficiently compact planetary systems. It may be torqued by planets so far inward that turbulence driven by the magneto-rotational instability, activated in the innermost regions which are sufficiently thermally ionized (e.g., Desch \& Turner 2015), takes over the job of disk accretion onto the host star. Or it may be torqued by planets so far outward that it escapes from the system altogether in a photoionized wind (e.g., Alexander et al. 2014).

How massive a gas disk can a set of super-Earths drain? A first consideration is that the disk surface density can not be so large that the embedded planet mass $M_{\mathrm{p}}<M_{\mathrm{cr}} \propto \Sigma_{\mathrm{p}}^{5 / 13}$, lest the planet migrate away. Based on the typical parameters listed in Equation 1, a disk containing $\Sigma_{\mathrm{p}} r_{\mathrm{p}}^{2} \sim 10^{-3} M_{*} \sim$ $300 M_{\oplus}$ of gas could be evacuated by $\sim 6$ super-Earths weighing a total of $\sim 30 M_{\oplus}$. In such an initially gas-rich environment, we anticipate the planets would migrate to and fro by a few tens of percent in orbital distance (Figure 1). As the disk drains in the long term, whatever slow and erratic migration the planets undergo diminishes. Most of the angular momentum of interplanetary gas would be transported to the outermost disk, exterior to all the planets, either by Lindblad torques or by direct advection.

The large cavities of transitional disks may have been excavated over time by families of super-Earths. Observationally, gas densities inside cavities can be suppressed relative to their values outside by two to four orders of magnitude (e.g., Carmona et al. 2017, Dong et al. 2017). To reproduce these strong depletions, appeal is commonly made to giant planets in viscous $\alpha$-disks that can open deep gaps (e.g., Dong \& Dawson 2016, Dong \& Fung 2017). But an alternative interpretation is that the cavities have been eroded gradually over time by much smaller mass planets in inviscid disks. So far as we have measured in our simulations, such planets open gaps having only order-unity surface density contrasts. Nevertheless, given sufficient time, they can drain interplanetary gas by orders of magnitude. Because planet-driven accretion rates $\dot{M}$ scale linearly with gas surface densities $\Sigma$, we have $\dot{\Sigma} \propto-\Sigma$ which implies exponential decay of the gas content. If it takes $t_{\mathrm{e}-\text { fold }} \sim 10^{5} \mathrm{yr}$ to reduce a total disk mass of $10^{-3} M_{\odot}$ by a factor of $e$ (this assumes a contemporaneous mass transport rate of $\left.10^{-8} M_{\odot} \mathrm{yr}^{-1}\right){ }^{2}$ then it takes $7 t_{\mathrm{e}-\text { fold }} \sim 7 \times 10^{5} \mathrm{yr}$ to reduce it by a factor of 1000 . Another feature of this picture is that if super-Earths drain disk mass faster than it takes for their nascent atmospheres to cool and acquire more mass, they may be able to forestall runaway accretion (Lee et al. 2014). Depleting the local disk density by a factor of $\gtrsim 100$ (relative to the minimum-mass extrasolar nebula) over timescales of $\sim 1$ Myr suffices to keep the gas-to-solids mass fraction of super-Earths $\lesssim 10 \%$, in accord with observations (Lee \& Chiang 2016).

One potential problem with this scenario is that it predicts mass accretion rates to lower in proportion to disk gas densities. Although some transitional disks do have low accretion rates (e.g., Dong et al. 2017), others do not, with a few having $\dot{M}$ as high as $10^{-7} M_{\odot} \mathrm{yr}^{-1}$ (e.g., Rosenfeld et al. 2014, Carmona et al. 2017, Wang \& Goodman 2017). Even so, system-to-system variations in orbital architectures, particularly in the masses of the orbiting companions, could help to resolve this problem. We have focused here on super-Earths because they are commonplace, but in principle gas giants and perhaps brown dwarfs or even low-mass stars may serve in their stead 3

\footnotetext{
${ }^{2}$ This transport can be inward or outward-it should not matter as long as the region occupied by the planets is monotonically drained of gas over time.

${ }^{3}$ The case of the transitional disk HD 142527 is especially intriguing: its host star accretes at a rate of $\dot{M} \sim 10^{-7} M_{\odot} \mathrm{yr}^{-1}$ Garcia Lopez et al. 2006), and its cavity contains a $0.2 M_{\odot}$ companion highly inclined to the disk (Casassus et al. 2015. Lacour et al. 2016).
} 
That disk gas is intrinsically inviscid (laminar) is suggested on other, independent grounds. Large-scale asymmetries in transitional disks (e.g., Casassus et al. 2013, van der Marel et al. 2013; (Pinilla et al. 2015) have been interpreted as vortices (e.g., Zhu \& Baruteau 2016, Baruteau \& Zhu 2016), but these vortices are spawned only in low-viscosity disks. Zhu $\&$ Baruteau (2016) found that the Shakura-Sunyaev viscosity parameter $\alpha$ needed to be $10^{-4}$ or lower before vortices could grow from sharp density gradients. In other news, attempts to detect turbulence in the outer portions of disks using molecular line observations have so far come up emptyhanded (Flaherty et al. 2015; Flaherty et al. 2017, in preparation). And Rafikov (2017), in a systematic analysis of disk accretion rates and masses, suggests that accretion may not proceed viscously (i.e., diffusively), but may be enabled instead by spiral density waves and/or disk winds. All these recent developments, in addition to our present work, suggest that the reason the community has not discovered a robust explanation for a non-zero $\alpha$ in protoplanetary disks is that none exists: that in fact such disks are for the most part inviscid, and accrete primarily by the action of gravitational torques, exerted either by disk gas itself at early times (e.g., Gammie 2001; Cossins et al. 2009) ${ }^{4}$ or by planets at late times.

We thank Pawel Artymowicz, Ruobing Dong, Eve Lee, Hui Li, Zhi-Yun Li, Frédéric Masset, Norm Murray, Ruth Murray-Clay, Sijme-Jan Paardekooper, Roman Rafikov, Yanqin $\mathrm{Wu}$, Zhaohuan $\mathrm{Zhu}$, and an anonymous referee for encouraging discussions and helpful feedback. This work was performed under contract with the Jet Propulsion Laboratory (JPL) funded by NASA through the Sagan Fellowship Program executed by the NASA Exoplanet Science Institute. EC is grateful for financial support from NASA and NSF.

\section{REFERENCES}

Alexander, R., Pascucci, I., Andrews, S., Armitage, P., \& Cieza, L. 2014, Protostars and Planets VI, 475

Bai, X.-N. 2016, ApJ, 821, 80

Baruteau, C., \& Zhu, Z. 2016, MNRAS, 458, 3927

Burke, C. J., Christiansen, J. L., Mullally, F., et al. 2015, ApJ, 809, 8

Calvet, N., D’Alessio, P., Watson, D. M., et al. 2005, ApJL, 630, L185

Carmona, A., Thi, W. F., Kamp, I., et al. 2017, A\&A, 598, A118

Casassus, S., van der Plas, G., M, S. P., et al. 2013, Nature, 493, 191

Casassus, S., Marino, S., Pérez, S., et al. 2015, ApJ, 811, 92

Chiang, E., \& Laughlin, G. 2013, MNRAS, 431, 3444

Christiansen, J. L., Clarke, B. D., Burke, C. J., et al. 2015, ApJ, 810, 95

Clanton, C., \& Gaudi, B. S. 2014, ApJ, 791, 91

Colella, P., \& Woodward, P. R. 1984, Journal of Computational Physics, 54, 174

Cossins, P., Lodato, G., \& Clarke, C. J. 2009, MNRAS, 393, 1157

Cumming, A., Butler, R. P., Marcy, G. W., et al. 2008, PASP, 120, 531

D'Angelo, G., \& Lubow, S. H. 2010, ApJ, 724, 730

Desch, S. J., \& Turner, N. J. 2015, ApJ, 811, 156

Dong, R., \& Dawson, R. 2016, ApJ, 825, 77

Dong, R., \& Fung, J. 2017, ApJ, 835, 146

Dong, R., van der Marel, N., Hashimoto, J., et al. 2017, ApJ, 836, 201

Dressing, C. D., \& Charbonneau, D. 2015, ApJ, 807, 45

Flaherty, K. M., Hughes, A. M., Rosenfeld, K. A., et al. 2015, ApJ, 813, 99

Fressin, F., Torres, G., Charbonneau, D., et al. 2013, ApJ, 766, 81

Fung, J. 2015, PhD thesis, University of Toronto, Canada

Fung, J., \& Chiang, E. 2016, ApJ, 832, 105

Fung, J., Masset, F., Lega, E., \& Velasco, D. 2017, AJ, 153, 124

Fung, J., Shi, J.-M., \& Chiang, E. 2014, ApJ, 782, 88

Gammie, C. F. 2001, ApJ, 553, 174

Garcia Lopez, R., Natta, A., Testi, L., \& Habart, E. 2006, A\&A, 459, 837

Goodman, J., \& Rafikov, R. R. 2001, ApJ, 552, 793
Hartmann, L., D’Alessio, P., Calvet, N., \& Muzerolle, J. 2006, ApJ, 648, 484 Hourigan, K., \& Ward, W. R. 1984, Icar, 60, 29

Ingleby, L., Calvet, N., Herczeg, G., et al. 2013, ApJ, 767, 112

Kley, W., \& Nelson, R. P. 2012, ARA\&A, 50, 211

Lacour, S., Biller, B., Cheetham, A., et al. 2016, A\&A, 590, A90

Lee, E. J., \& Chiang, E. 2016, ApJ, 817, 90

Lee, E. J., Chiang, E., \& Ormel, C. W. 2014, ApJ, 797, 95

Li, H., Lubow, S. H., Li, S., \& Lin, D. N. C. 2009, ApJL, 690, L52

Lin, M.-K., \& Papaloizou, J. C. B. 2010, MNRAS, 405, 1473

Malik, M., Meru, F., Mayer, L., \& Meyer, M. 2015, ApJ, 802, 56

Masset, F. S., \& Papaloizou, J. C. B. 2003, ApJ, 588, 494

Müller, T. W. A., Kley, W., \& Meru, F. 2012, A\&A, 541, A123

Paardekooper, S.-J., \& Papaloizou, J. C. B. 2009, MNRAS, 394, 2297

Peplinski, A. 2008, PhD thesis, University of Stockholm

Pinilla, P., van der Marel, N., Pérez, L. M., et al. 2015, A\&A, 584, A16

$\mathrm{Pu}, \mathrm{B} .$, \& Wu, Y. 2015, ApJ, 807, 44

Rafikov, R. R. 2002a, ApJ, 569, 997

-. 2002b, ApJ, 572, 566

—. 2017, ApJ, 837, 163

Rosenfeld, K. A., Chiang, E., \& Andrews, S. M. 2014, ApJ, 782, 62

Sari, R., \& Goldreich, P. 2004, ApJL, 606, L77

Sicilia-Aguilar, A., Henning, T., \& Hartmann, L. W. 2010, ApJ, 710, 597

Tsang, D., Turner, N. J., \& Cumming, A. 2014, ApJ, 782, 113

van der Marel, N., van Dishoeck, E. F., Bruderer, S., et al. 2013, Science, 340,1199

Wang, L., \& Goodman, J. J. 2017, ApJ, 835, 59

Ward, W. R., \& Hourigan, K. 1989, ApJ, 347, 490

Yu, C., Li, H., Li, S., Lubow, S. H., \& Lin, D. N. C. 2010, ApJ, 712, 198

Zhu, Z., \& Baruteau, C. 2016, MNRAS, 458, 3918

\footnotetext{
${ }^{4}$ In self-gravitating disks, characterizing transport in terms of a non-zero $\alpha$ is commonly done, but mostly for convenience. Gravity is a long-range
} 